Musées, Patrimoine et Culture scientifiques et techniques

182 | 2019

mars-avril 2019

\title{
Journal de bord d'un parcours multi-sensoriel
}

\section{Caroline Jules}

\section{OpenEdition \\ Journals}

Édition électronique

URL : http://journals.openedition.org/ocim/2349

DOI : $10.4000 /$ ocim. 2349

ISSN : 2108-646X

Éditeur

OCIM

Édition imprimée

Date de publication : 1 mars 2019

Pagination : $34-41$

ISSN : 0994-1908

Référence électronique

Caroline Jules, « Journal de bord d'un parcours multi-sensoriel », La Lettre de I'OCIM [En ligne], 182 |

2019, mis en ligne le 01 mars 2020, consulté le 17 mars 2020. URL : http://journals.openedition.org/ ocim/2349; DOI : https://doi.org/10.4000/ocim.2349

Ce document a été généré automatiquement le 17 mars 2020.

Tous droits réservés 


\section{Journal de bord d'un parcours multi-sensoriel}

\section{Caroline Jules}

Reproduction du buste de Sainte-Anastasie

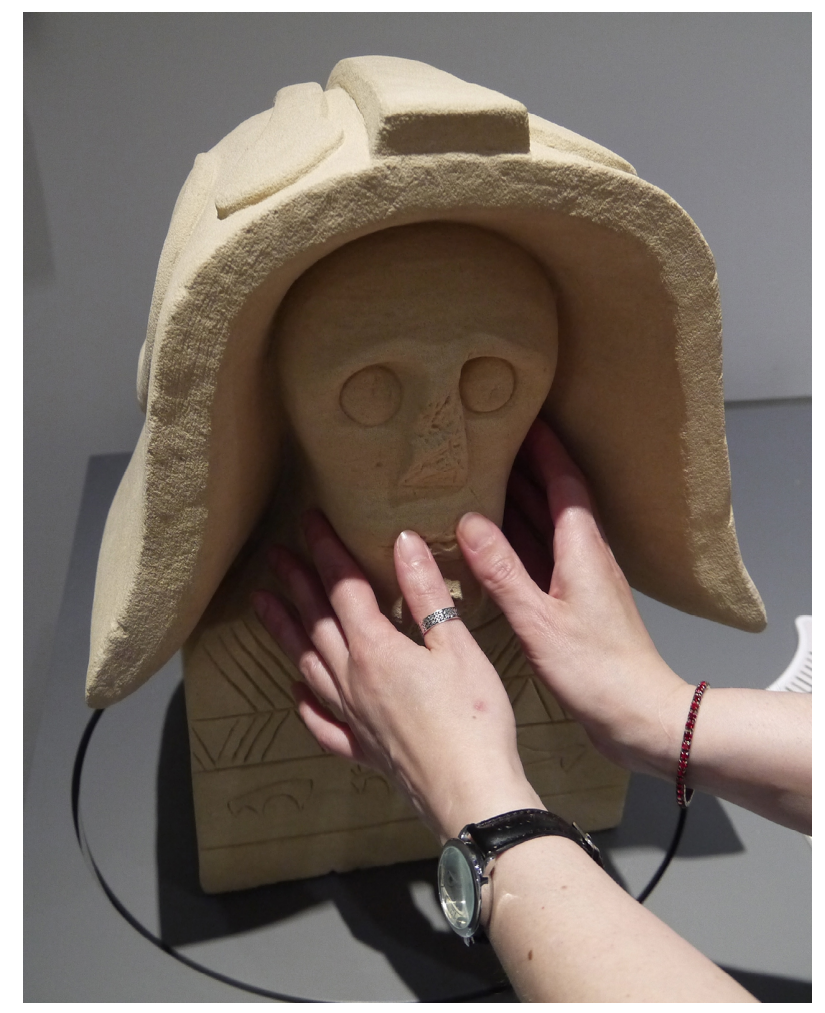

() Musée de la Romanité. Ville de Nîmes/ Tactile Studio 


\section{Pourquoi proposer un parcours multi-sensoriel ? (début 2017)}

\section{Au-delà de la loi}

1 Depuis la loi du 11 février 2005 pour «la participation et la citoyenneté des personnes handicapées", les établissements recevant du public doivent être accessibles à tous. Ainsi, les musées, de plus en plus souvent, conçoivent des solutions techniques, technologiques et humaines pour l'accès aux espaces et aux offres culturelles.

2 Au musée de la Romanité, l'architecture et la scénographie, confiées à l'agence Elizabeth de Portzamparc (la maîtrise d'œuvre), avaient été pensées dès l'origine en respect des normes d'accessibilité. On y trouve, par exemple, une bande de guidage au sol pour rejoindre l'accueil, des alarmes visuelles dans les toilettes pour les personnes sourdes...

3 Cependant, ce n'est que début 2017 que l'idée du parcours multi-sensoriel a germé dans l'esprit du personnel du musée (la maîtrise d'ouvrage), en accord avec l'agence Portzamparc. Était-ce une obligation légale? Non. Mais alors, pourquoi concevoir un tel projet?

\section{Des objectifs pour tous les publics}

4 Sans se limiter à un parcours tactile pour les personnes déficientes visuelles, l'intérêt d'un tel parcours est de solliciter plusieurs sens et de s'adresser à divers publics. À l'heure où l'intérêt pour les neurosciences et les intelligences multiples se démocratise, il convient donc de penser la découverte et l'apprentissage selon les envies et les compétences de chacun. Ainsi, à Nîmes, les objectifs de médiation étaient nombreux :

5 - rendre accessible le musée aux publics handicapés visuels, mentaux, moteurs et auditifs ;

6 - viser d'autres publics, notamment les familles, avec des envies et des compétences différentes liées à l'âge, au niveau de lecture, au niveau de compréhension, à l'attention, à la prédominance d'un sens sur un autre... ;

7 - concevoir un parcours de médiation multi-sensoriel avec des éléments visuels, tactiles et sonores ;

8 - proposer une approche pédagogique, ludique et sensorielle ;

9 - concevoir des outils de médiation qui ne soient pas une "simplification" des contenus scientifiques mais qui apportent un complément d'information pour tous les visiteurs ;

10 - réaliser un parcours répondant aux attentes de l'accessibilité universelle (« design for all »), c'est-à-dire facile à comprendre et ergonomique.

11 D'un point de vue matériel, il fallait également :

12 - rendre ce parcours accessible en visite libre et en individuel, mais également utilisable avec un médiateur; 
13 - aborder à la fois l'architecture du musée, mais aussi toutes les périodes historiques (Protohistoire, Antiquité, Moyen-Âge) et les principaux thèmes des collections (vie quotidienne, religion, artisanat...) ;

14 - respecter l'esthétique générale du musée ;

15 - viser l'obtention de la marque « Tourisme et Handicap »;

16 - répartir le parcours tout au long de la visite pour une intégration totale des publics spécifiques au sein du grand public ;

17 - proposer une découverte des objets de différentes manières: usage, forme, iconographie, matière, échelle, histoire, comparaison ;

18 - augmenter la visibilité du musée auprès des personnes handicapées et des familles.

19 La volonté d'un tel parcours s'inspirait d'exemples réussis. Citons, par exemple, les parcours du Mucem à Marseille (13), du musée de l'Homme à Paris (75), du musée de Flandre à Cassel (59)...

\section{La conception du parcours (printemps-été 2017)}

\section{Constituer une équipe}

20 À Nîmes, avant l'été 2017, l'équipe du musée a contacté Culture accessible pour se faire aider. Culture accessible a donc été chargée de l'assistance à maîtrise d'ouvrage (Amo).

21 Il a alors fallu franchir des phases de conception et des étapes administratives. Quels que soient la taille du lieu et son statut, dès la phase de conception, il convient effectivement d'avoir en tête tous les services à solliciter à divers moments du projet, parmi lesquels :

22 - l'équipe scientifique (service conservation et service médiation);

23 - l'Amo pour suivre l'intégralité du projet ;

24 - l'agence de scénographie Portzamparc, qui a assuré le respect de l'esthétique, la localisation des éléments tactiles et la conception du mobilier ;

25 - le service de la commande publique pour le passage de l'appel d'offres ;

26 - le fournisseur des outils multimédias.

\section{Définir le contenu du parcours...}




\section{... et son emplacement}

Tous ces outils doivent bien sûr trouver leur place dans le musée. Cela nécessite une collaboration étroite avec l'équipe scénographique qui a validé l'emplacement des modules multi-sensoriels sur les plans et réalisé le dessin des mobiliers. Le fabricant des mobiliers et le socleur ont également eu leur rôle à jouer.

Des bandes podotactiles mènent le visiteur malvoyant jusqu'à l'accueil du musée.

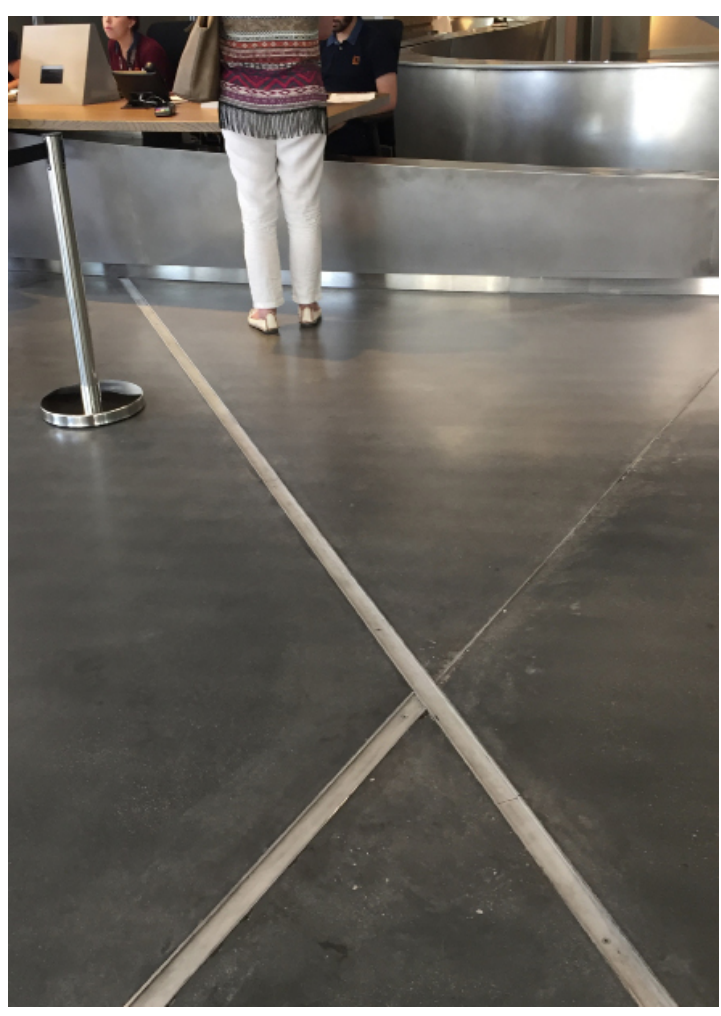

(c) C. Jules / Agence Elizabeth de Portzamparc

Maquette tactile du musée dans son quartier

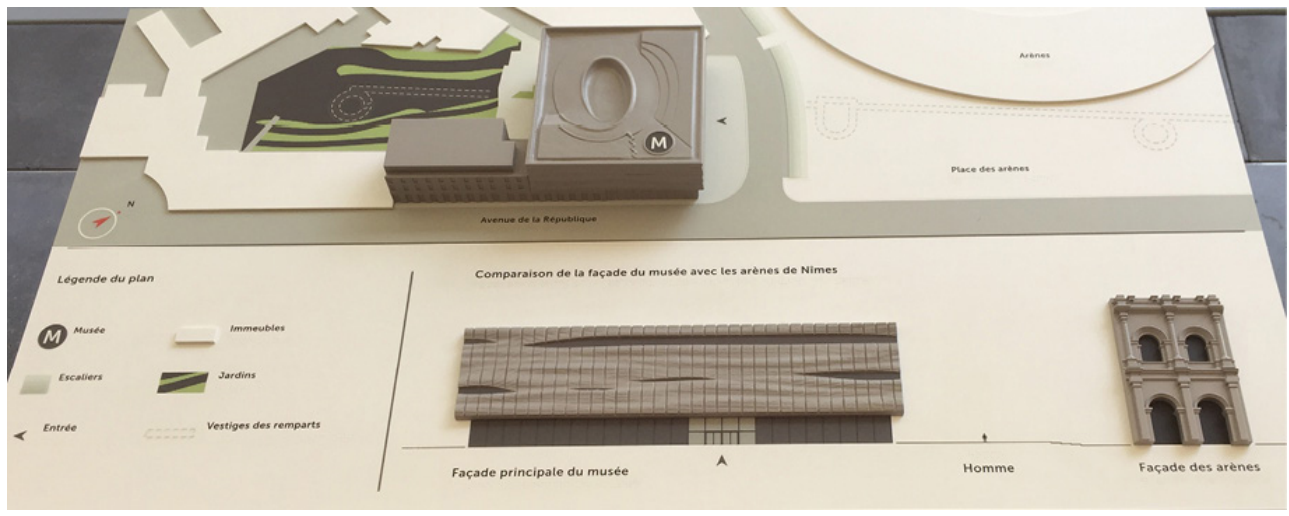

(c) C. Jules/Tactile Studio 


\section{L'appel d'offres (automne 2017)}

Dès qu'un projet dépasse un certain montant, les collectivités doivent passer un appel d'offres. Pour ce faire, le service de la commande publique de la collectivité doit être associé. Il faut aussi se demander si l'appel d'offres fera l'objet d'un seul lot ou de plusieurs. Pour répondre à cette question, il faut donc déjà avoir une idée des compétences des fournisseurs potentiels. Par exemple, dans le cas d'un parcours multisensoriel, en plus des objets à toucher, il peut y avoir des multimédias, la construction de tables et le soclage d'objets. Il y a donc peu de chance pour qu'un seul lot, c'est-àdire un seul fournisseur, suffise, même si ce dernier sous-traite quelques réalisations.

À Nîmes, le suivi administratif et le financement étaient assurés par la Mission Grands Projets. C'est donc avec ce service que les documents nécessaires à la mise en concurrence ont été produits. Parmi ces documents, certains sont purement administratifs. D'autres nécessitent d'y injecter des contenus qui font appel aux compétences du musée ou de l'Amo, tels que :

- le cahier des clauses techniques particulières (ССTP), sorte de cahier des charges qui reprend tout ce qui devra être fabriqué pour le parcours multi-sensoriel ;

- le règlement de consultation ( $\mathrm{RC}$ ), document qui liste les renseignements que le candidat devra fournir. Il s'agit, d'une part, des «pièces de la candidature » avec références professionnelles et capacités techniques de l'entreprise. D'autre part, les «pièces de l'offre » peuvent inclure une présentation de l'équipe, les matériaux des futures réalisations, les soclages prévus, les adaptations graphiques des images tactiles ;

- un calendrier prévisionnel à détailler par les postulants ;

- la décomposition du prix global et forfaitaire (DPGF) avec la désignation des ouvrages, le prix à l'unité et la quantité ;

42 - des annexes : celles-ci peuvent comprendre les plans du bâtiment, des visuels des œuvres à reproduire ou à commenter, la liste de ces œuvres, les dessins des futurs mobiliers s'ils ont déjà été esquissés, les plans d'implantation des modules multisensoriels...

Une fois les réponses reçues, le choix du candidat n'est pas chose aisée. Pour être le plus objectif possible, il convient, dans le RC, d'avoir listé des critères associés à des points. Cet exercice périlleux doit être le plus impartial possible, d'où l'importance de demander des pièces de l'offre cadrées, donc très comparables. L'Amo doit alors rédiger un rapport d'analyse des offres (Rao) et faire correspondre une note à chaque critère. À Nîmes, c'est l'entreprise Tactile Studio qui a été retenue.

Extrait du cahier des clauses techniques particulières (CCTP) de l'appel d'offres pour la fabrication des objets du parcours

Le CCTP doit être précis afin de faire comprendre au prestataire les attentes de la maîtrise d'ouvrage et de ne pas avoir de mauvaises surprises. Voici quelques exemples de ce qui peut y être mentionné.

La prestation doit inclure :

- les échanges avec les équipes du musée, le scénographe et le chargé de mission « accessibilité »; 
- la coordination du titulaire avec ses éventuels sous-traitants ;

- la définition des partis pris techniques et esthétiques pour la réalisation des objets ;

- la réalisation de versions d'essai pour un test ;

- la présence des titulaires pendant le test auprès de personnes en situation de handicap;

- la fourniture de plusieurs bons à tirer avant fabrication ;

- la fabrication et le suivi de fabrication des objets ;

- le soclage ;

- la collaboration si nécessaire avec les autres prestataires qui réalisent les parcours multimédias, les mobiliers et le soclage des objets ;

- le transport, le déballage et le montage ;

- les tests de bon fonctionnement sur place et la maintenance pendant le premier mois suivant l'installation du parcours ;

- la fourniture du DOE (dossier d'ouvrage exécuté).

Contraintes esthétiques

Le parcours devra être esthétique et s'intégrer dans l'esthétique générale du musée, au niveau de sa muséographie et au niveau graphique.

Contraintes techniques des objets

Les objets présentés devront :

- être installés de manière fixe ;

- être faciles à comprendre et ergonomiques ;

- respecter les normes de sécurité ERP avec des matériaux classés M1 (ignifugés et xylophagés) ;

- respecter les normes de conservation préventive (matériaux stables et neutres chimiquement, inorganiques) ;

- être solides pour une manipulation fréquente ;

- être non coupants ; 


\section{La fabrication et les tests (janvier-mai 2018)}

\section{Les choix graphiques}

Avant la reproduction d'une image ou d'une sculpture, une adaptation graphique doit être réalisée. Cela consiste en un choix de détails à garder, à grossir ou à supprimer. Il faut même parfois «tricher " pour éviter des détails trop petits pour l'œil ou la main, ou des perspectives difficiles à concevoir. En effet, des éléments tactiles ne sont pas des reproductions d'œuvres originales mais des interprétations. Cette phase assez longue nécessite donc une très bonne connaissance de la perception haptique (par le toucher) des publics déficients visuels.

\section{L'évaluation en cours de réalisation}

En avril 2018, des prototypes de certains outils tactiles ont été soumis à des personnes malvoyantes et non-voyantes. Deux réunions de test ont été organisées : une à Paris avec l'association Valentin Haüy (AVH) et l'autre à Nîmes avec l'antenne gardoise de la Fédération des aveugles de France (FAF 30). Ces séances de travail indispensables ont fait ressortir des remarques telles que la complexité de certains détails, les reliefs à accentuer... En effet, bien que les techniques choisies aient déjà fait leurs preuves, chaque projet constitue tout de même une nouvelle prouesse. Quel juste milieu trouver entre des documents simplistes ou trop complexes ? Comment satisfaire à la fois des habitués des images tactiles et des novices?

Module tactile du médaillon de Cavillargues

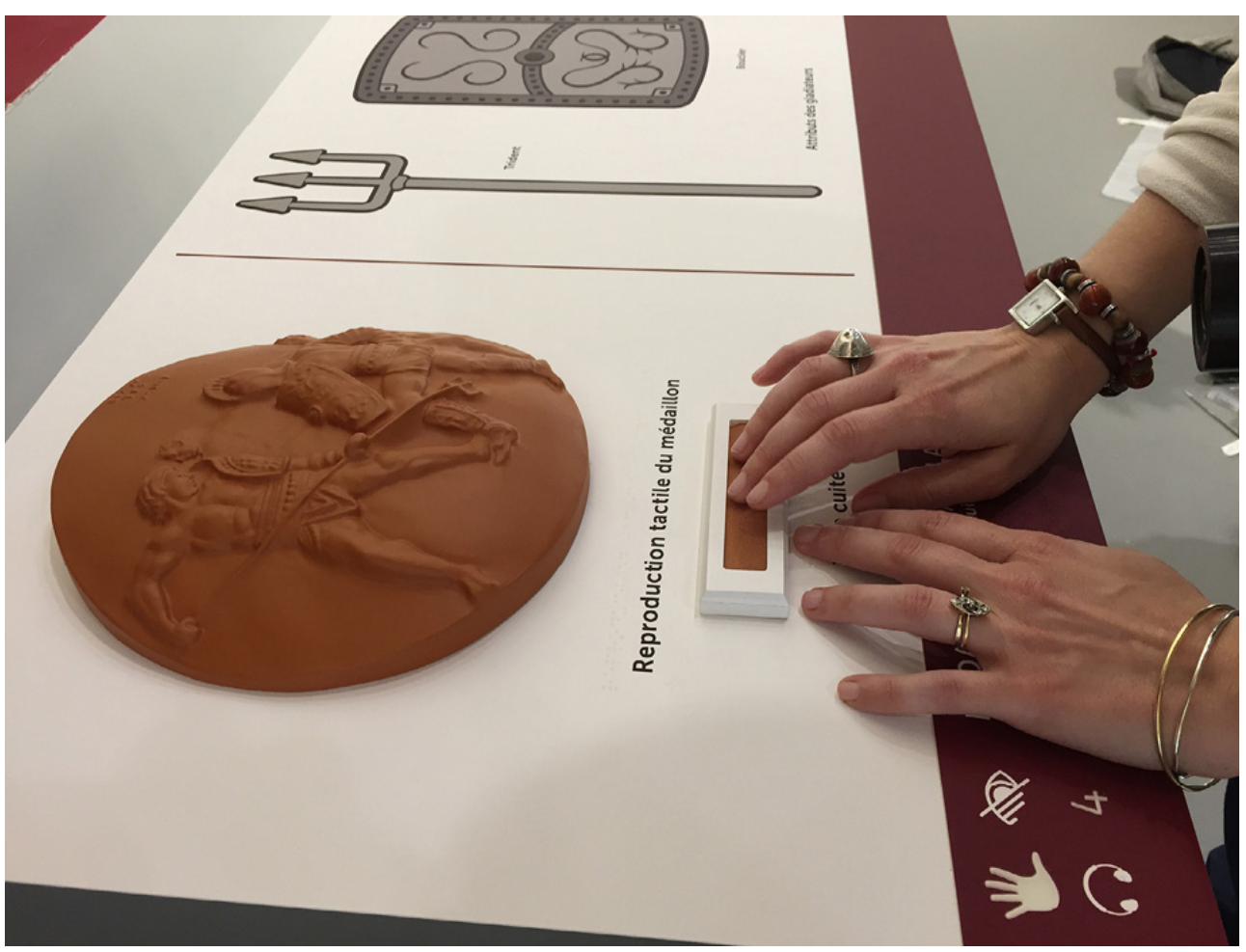

(C) C. Jules/Tactile Studio 


\section{L'installation et la finalisation (mai 2018)}

Pour les images et objets tactiles, les validations définitives se décident avec des visuels numérisés des futurs éléments. Il y a donc forcément une différence entre les versions bidimensionnelles sur écran et les réalisations tactiles qui suivront. Les 2D permettent cependant de bien comprendre le futur objet pour signer les bons à tirer.

Une fois fabriqués, les objets doivent être soclés sur le mobilier prévu à cet effet. À ce stade, une collaboration étroite avec le fabricant de mobilier et le socleur est indispensable. Le soclage d'éléments tactiles est toujours une opération délicate dans la mesure où il doit être très solide pour la manipulation et le plus discret possible au toucher. Ce soclage doit donc être prévu dès la phase de conception graphique de l'objet. Pour les reproductions de sculptures ou de maquettes, il convient ainsi souvent d'insérer des tiges filetées en dessous des objets au moment de la fabrication.

Une fois tous les objets installés, l'Amo doit vérifier qu'ils sont conformes à l'appel d'offres. Si certains objets ne conviennent pas, il faut alors émettre des réserves. Dans tous les cas, l'entreprise doit fournir un dossier d'ouvrage exécuté (DOE) avec la liste des opérations de maintenance à accomplir si besoin, les techniques d'entretien des objets et les coordonnées des sous-traitants éventuels.

Enfin, le musée doit mettre à disposition des publics handicapés, à l'accueil, un registre d'accessibilité. Ce document, obligatoire depuis 2017, inclut notamment une fiche qui résume ce qui est accessible ou non, ainsi qu'un guide pratique pour l'accueil.

Menu du visio-guide avec tous les parcours

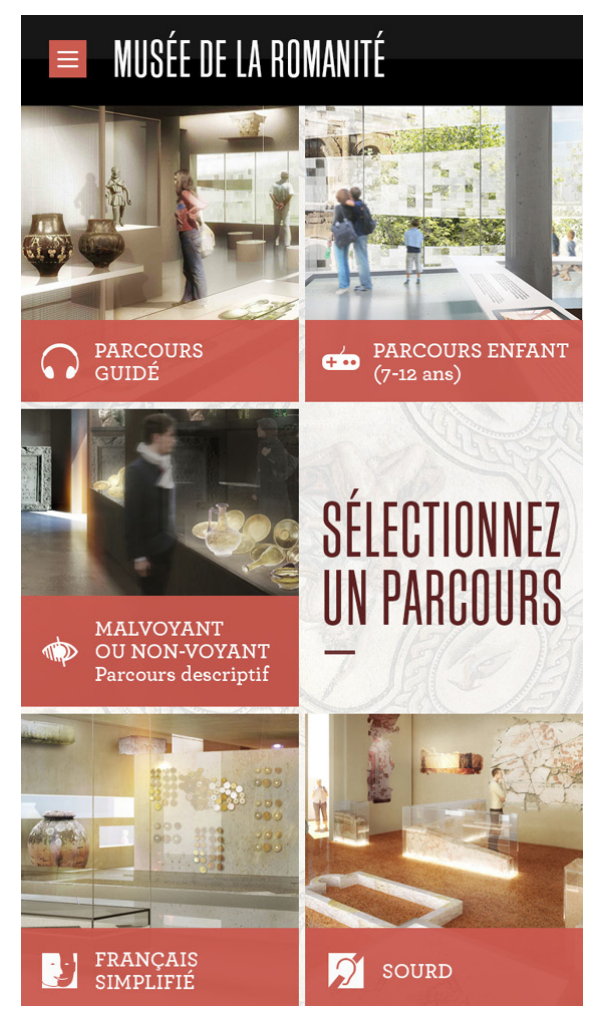

(c) Opixido, Ville de Nîmes 


\section{Les parcours multimédias adaptés à chaque besoin (été - automne 2018)}

Les outils visuels et tactiles ne suffisent pas toujours. Ainsi, les personnes aveugles ne comprennent pas ce qu'elles touchent sans un commentaire descriptif. De même, certains objets sortis de leur contexte ne sont pas reconnus par le public déficient intellectuel. Il convient de prévoir des aides multimédias.

51 À Nîmes, en plus des parcours multimédias adulte "grand public » et « enfant ", trois parcours ont été prévus pour les publics handicapés. C'est la société Opixido qui était chargée de ce marché. Précisons que le déclenchement des plages sonores se fait par des iBeacons. Cela signifie que la tablette prêtée à l'accueil vibre lorsque l'on se trouve à proximité d'un objet ayant un commentaire. Revenons donc sur ces trois parcours :

52 - un parcours audio-descriptif permet aux visiteurs déficients visuels d'avoir une description de chaque élément tactile. Cette description très détaillée suit le cheminement du doigt. Ce parcours se compose ainsi de treize plages sonores. À la fin de chaque plage, une indication de déplacement est donnée pour information mais elle ne permet pas un déplacement en autonomie. Ce parcours a été rédigé par Polymorphe Design (Maud Dupuis) pour Opixido ;

53 - un parcours en « français facile ». Il s'agit de descriptions simplifiées d'objets pour les personnes handicapées mentales. Ce texte a été rédigé par Atelier Culture (Amandine Greilich) pour Opixido. Il se compose de treize plages sonores également ;

54 - un parcours en Langue des signes française. Ce parcours a été enregistré par Langue Turquoise pour Opixido.

Parmi les étapes de conception de ces parcours, il faut inclure une phase de test, qui consiste en une séance d'écoute des plages sonores par des personnes déficientes visuelles et, à un autre moment, par des personnes déficientes intellectuelles. Il n'est pas conseillé de faire l'économie de cette phase dans la mesure où il en ressort souvent un besoin d'effectuer des modifications. Enfin, avant l'enregistrement par des comédiens professionnels, une relecture par l'équipe scientifique doit valider la véracité scientifique des contenus.

\section{Plage sonore en français simplifié de l'« As de Nîmes "}

Vous regardez et pouvez même toucher une pièce de monnaie de l'époque des Romains au fond de l'espace. Elle est beaucoup plus grande que la vraie pièce. La vraie pièce est dans la petite vitrine derrière vous au milieu.

Les billets, les chèques, les cartes bleues n'existaient pas. On payait avec des pièces qui avaient des valeurs différentes.

La pièce que vous regardez est appelée l'as de Nîmes, mais c'est un dupondius. Le dupondius est une monnaie comme l'euro aujourd'hui. Et on dit l'as de Nîmes car elle a été fabriquée à Nîmes.

Regardez les deux faces de la pièce. Sur la première face, à gauche, on voit deux visages de profil, c'est-à-dire vu de côté. Il s'agit de deux personnes connues de l'époque. 
Le premier personnage porte une couronne avec des feuilles de laurier. Le laurier est une plante dont les feuilles sont un peu ovales et pointues au bout. Celui qui portait cette couronne était l'empereur de Rome, c'est-à-dire le grand chef. Son nom est Auguste. C'est le fils de Jules César. On le sait grâce à la couronne mais aussi grâce aux lettres IMP DIVI F (lire les lettres une par une) qui veut dire " Empereur fils du divin ». L'empereur, c'est le chef des Romains. Le fils du divin veut dire qu'il est le fils de Jules César.

Le deuxième personnage s'appelle Agrippa. Il porte une couronne en forme de proue de bateau. La proue est la partie avant d'un bateau. Cela veut dire qu'Agrippa a eu cette couronne pour le récompenser d'avoir gagné une bataille importante sur la mer.

Cette bataille est celle d'Actium. Actium est une ville en Grèce. La bataille opposait les Romains contre les Égyptiens. Ce sont donc les Romains qui ont gagné. Les Égyptiens vivent en Égypte où se trouvent les pyramides. C'est Cléopâtre qui était leur chef.

Si l'on résume : cette première face de la pièce représente deux personnages : Auguste, le chef des Romains et Agrippa, celui qui a gagné la bataille contre l'Égypte.

Sur la deuxième face on voit un crocodile et une palme. Le crocodile est l'animal qui représente l'Égypte car il y a beaucoup de crocodiles là-bas. On voit que ce crocodile est attaché à une palme par une chaine. La palme est la feuille du palmier. Le palmier est un grand arbre avec de très grandes feuilles en forme de plume. La palme représente l'empire Romain.

Que signifie cette image ? Cela veut dire que l'Égypte, représentée par le crocodile, a perdu la bataille. L'Égypte appartient maintenant aux Romains, représentés par la palme. C'est pour ça que le crocodile est attaché avec une chaîne.

On voit des lettres : COL NEM (lire les lettres une par une). Cela veut dire colonie de Nîmes. Une colonie est une ville qui appartient à Rome.

Aujourd'hui encore on peut voir partout à Nîmes le palmier et le crocodile car c'est le symbole de la ville. C'est-à-dire que c'est le dessin qui représente la ville.

Amandine Greilich, consultante et formatrice en projets culturels, Atelier Culture 
Image tactile de la monnaie dite " As de Nîmes "

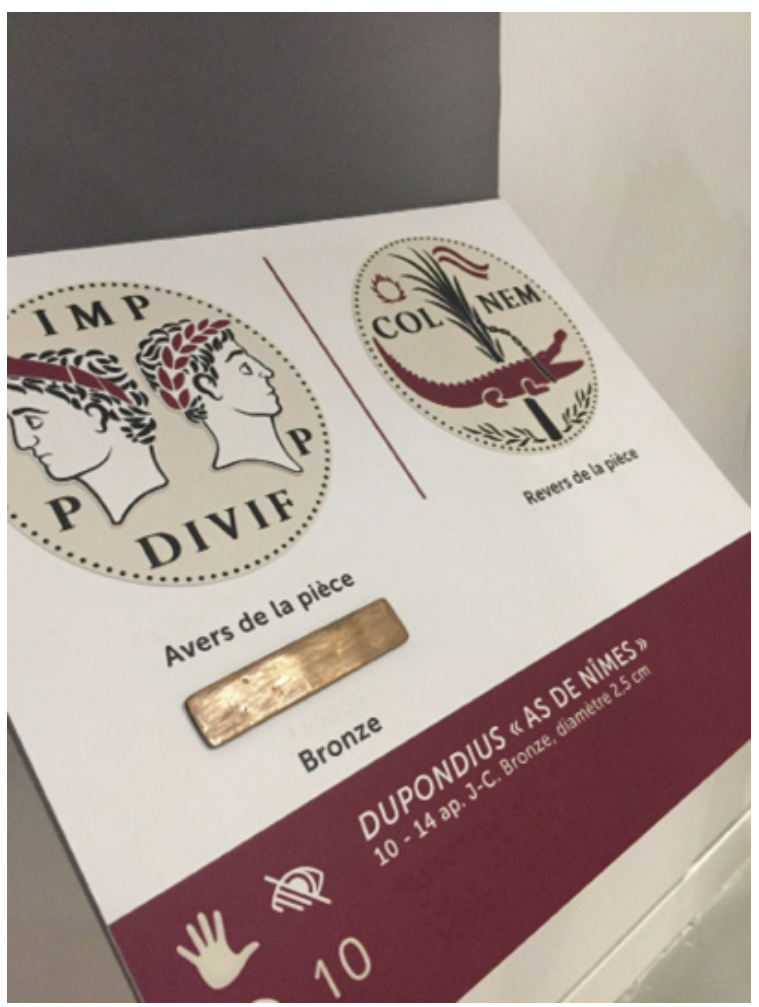

(c) C. Jules/Tactile Studio

\section{Un parcours pour tous}

Un parcours tactile nécessite donc une méthodologie globale qui doit mobiliser l'ensemble des services. Cela montre également à quel point l'accessibilité doit être clairement intégrée dans les projets culturels et scientifiques des établissements.

Pour trouver son public, d'autres phases doivent aussi être pensées en parallèle : la communication et l'évaluation. Tout projet culturel n'est rien s'il n'est pas porté à la connaissance du plus grand nombre. En plus de la communication interne, notamment auprès des agents d'accueil, un travail doit être fait auprès d'associations et d'établissements spécialisés par des brochures, des présentations orales, Internet, les réseaux sociaux... Un tel parcours peut également permettre d'espérer obtenir la marque " Tourisme et handicap ».

Avec ces parcours multi-sensoriels en accès libre, les musées jouent ainsi pleinement leur rôle social en misant sur l'intégration de nouveaux publics. 
Test du parcours sonore « Handicap mental »

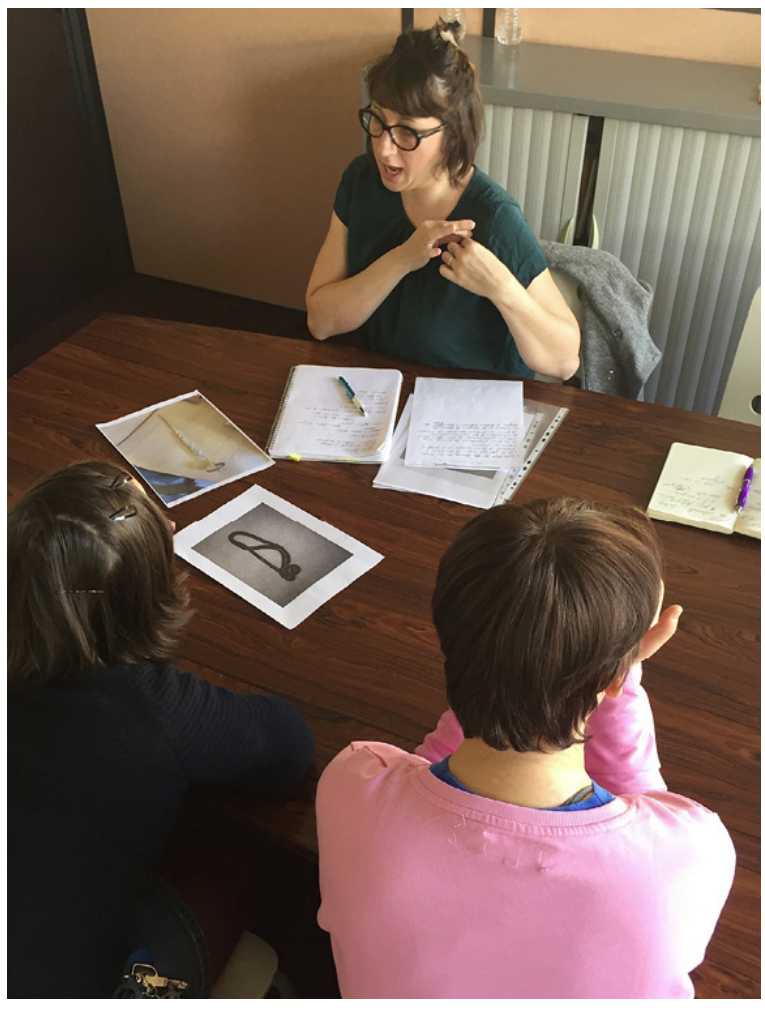

(C) C. Jules/A. Greilich/ESAT H. Rivet, Alged, Lyon

\section{BIBLIOGRAPHIE}

Collectif Expositions et parcours de visite accessibles. Ministère de la Culture et de la Communication, 2017.

Allaire, C. (dir.) Informer les personnes sourdes ou malentendantes. Éditions Inpes, 2012.

Gardner, H. Les intelligences multiples. Éditions Retz, collection Petit forum, 2004.

Informations sur la marque « Tourisme et handicap » : www.entreprises.gouv.fr/marques-

nationales-tourisme/marque-d-etat-tourisme-et-handicap

Liens vers des audiodescriptions en ligne sur des sites de musées :

Musée de Libourne :

www.ville-libourne.fr/mes-loisirs/le-musee/le-musee-des-beaux-arts/440-

audiodescriptions.html

Musée des Beaux-Arts de Quimper :

www.mbaq.fr/fr/visites/handicap/individuel-271.html

Musée d'Orsay, Paris : 
www.musee-orsay.fr/fr/espace-particuliers/particuliers/accessibilite/deficience-visuelle/ ressources/parcours-audiodescriptif.html?S=3

Musée des Beaux-Arts de Chambéry :

www.cultureaccessible.fr/pages/parcours-tactile-audiodescription/colloques-etinterventions-2.html

\section{RÉSUMÉS}

Afin de mettre en valeur son patrimoine romain, Nîmes a ouvert en juin dernier le musée de la Romanité doté d'un parcours multi-sensoriel. Pour le réaliser, quelles étapes ont dû être franchies? Avec quels interlocuteurs? À travers ce cas concret, c'est ici une méthodologie qui est proposée pour concevoir un parcours sensible, dans un lieu neuf ou en réaménagement.

INDEX

Mots-clés : Accessibilité, scénographie, handicap

\section{AUTEUR}

\section{CAROLINE JULES}

Caroline Jules est consultante en accessibilité culturelle contact@cultureaccessible.fr 